\title{
Research on Application of Sensors in Industrial Control Systems
}

\author{
HOU Wei ${ }^{1, a}$ \\ ${ }^{1}$ Chongqing College of Electronic Engineering, chongqing China, 401331 \\ a22258474@qq.com
}

Keywords: Sensors, Industrial Control, Wireless Sensor, System Design

\begin{abstract}
In the automation and control systems, sensor systems at the head, which is a direct effect on the measurement, and according to certain rules to be measured is converted into the same kind of money or other kinds of output, fast and accurate access to information and can withstand the harsh environment test, it is mechatronic systems achieve a high level of assurance. Without these sensors and system status information accurate and reliable automatic detection, information processing and decision-making functions of the system control can't be achieved. With the progress and development of sensor products, industrial monitoring and control system established by the sensor can solve problems that result from changes in the environment caused by uncertainties, thus reducing the production of wood products to improve work efficiency. Wireless sensor networks in the field of industrial control the development process, technical characteristics, common technology, application examples and problems should pay attention to choose to use are described.
\end{abstract}

\section{Introduction of Sensor Technology}

Application and development of industrial networks for industrial automation not only makes the low price, but also help improve the management and operation of industrial model to improve profitability. Industrial network is not limited to office and commercial environments, can also be extended to the manufacturing process, process monitoring and control, factory, or even the whole enterprise, there are a wide variety of industrial networks for enterprises to choose to install, sensor and actuator networks provide remote measurement, control and communicate and interact with the physical world, with a broad and deep potential applications in industrial automation.

Sensor means to measure the direct effect is, according to certain rules to be able to convert the same kind or other kinds of values output device. The sensor can be considered an extension of the human senses sensors are used to detect the automation system itself and the operation target operating environment state must provide relevant information for the effective control of mechatronic systems operations. The development of modern science and technology continue to put forward new requirements to sensor technology and promote the development of sensor technology at the same time, sensor technology and integrated quickly draw various scientific and technological fields (such as physics, chemistry, biology, etc.) of the new achievements, develop new the force method and apparatus for sensing technology has become an important field of modern technology, sensor systems and production has become an important emerging industries ${ }^{[1]}$.

With the development of new materials for sensors sensitive materials industry further development, the new sensor sensitive material has introduced high-tech materials are widely used in the manufacture of new sensors under development, development of new materials for the sensor industry plays a decisive role. With the development of modern, functional sensor has broken through the traditional functions, the output is no longer a single analog signal, but after digital signal processing microcomputer after a good, even with a little control, namely smart sensor to the micro power passive consumption and development: sensors are generally non-transformed to electricity consumption, the development of micro-power sensors and passive sensors is necessary to force development.

\section{Sensor Technological Advantage in Industrial Application}

Sensor technology is one of the important around the automation system (or product) development, being widely used in various automation products, robotics sensors through a variety of sensors can 
accurately perceive their own industrial robot, the operation target state and the working environment for robot control provides feedback information to enable it to operate accurately ${ }^{[2]}$. Fig.1 shows the technological advantage of sensor application.

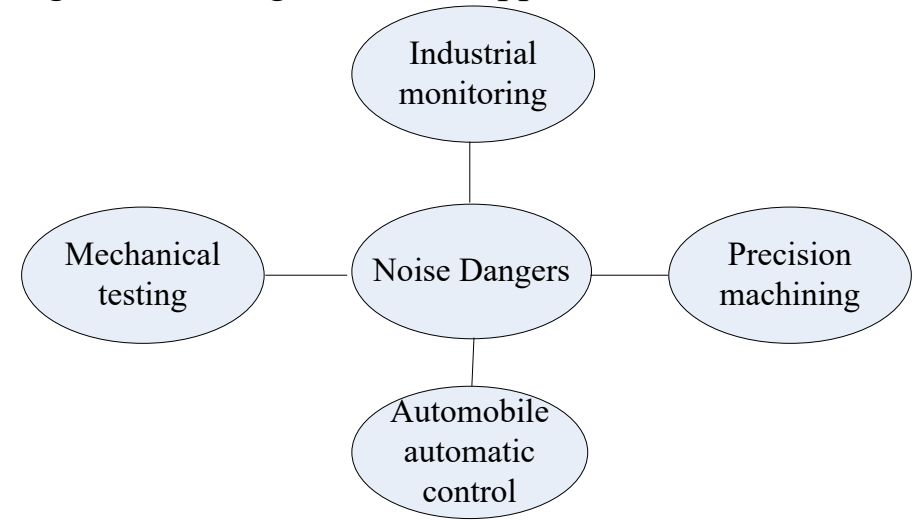

Fig. 1 The technological advantage of sensor application

Sensor technology is one of the key technologies of the production site will be fewer small can, in order to control the machine in accordance with the directive of cutting, need to use the sensor detects the relative displacement of the machine affect the position, speed and deformation, vibration and other factors on the tool parts, based on the detection result changes include tool change, etc., plus the conditions, in order to maintain the accuracy of plus, plus the realization of high precision cutting Furthermore, in order to achieve unmanned, labor-saving plus, also need to use sensors coupled with the detection results output feedback. Plus mechanical precision importance final plus, the final accuracy of plus size is the most important element is the straight line distance between two points connected to its base point is a position detection member on one point.

Typically use the touch sensor and the tiny points of light non-contact optical displacement sensor close to noon, it is possible to get a short time a large number of measurement data obtained closer to the actual situation, the high reliability of the measurement results, which is its biggest advantage in addition, tool management force, combined with use of an electromagnetic force type identification system to identify and carry out tool life management vehicle automatic control system with sensor technology electric cars off the small extent of the increase, the conventional mechanical system already is difficult to solve some of the issues related to the functional requirements of car, instead of the sensor is used as automotive electronic control electronic control system the key components of the system, which directly affect the technical performance of the car before the play, about an ordinary car is equipped with sensors 10-20, luxury cars are more, these sensors are mainly distributed in the engine control system, chassis control systems and vehicle control systems ${ }^{[3]}$.

\section{Wireless Sensor Applications}

Wireless sensor networks with low cost, easy to use and ubiquitous sensing and other characteristics, the traditional practice due to cost factors such as failure to achieve some important measurement line detection parameters of the industrial process becomes possible, thereby optimizing the implementation of control, improve product quality reduce energy efficiency, reduce maintenance costs run. The wireless technology is used in industrial automation field devices to detect and control, Instrument Society of America (I SA) to develop ISA 100 series of standards, including applications for industrial wireless network standards, the establishment of complete protocol architecture $^{[4]}$. Fig. 2 shows the structure of wireless sensor application. 


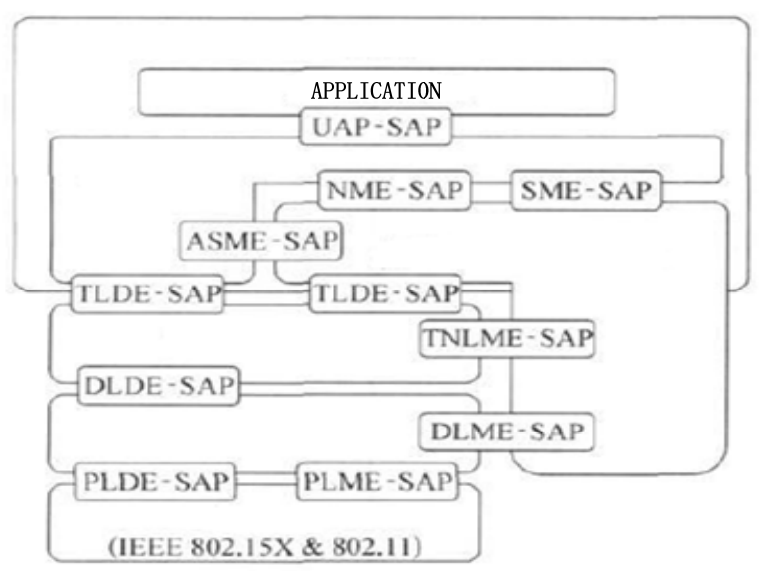

Fig. 2 The structure of wireless sensor application

Physical Layer: modulation is responsible for sending and receiving data. Data Link Layer: responsible for data framing, frame detection, medium access and error control. Network and transport layers: Industrial Wireless networks are usually two layers and a layer of consideration, complete end to end reliable data transfer of data. Network management: the completion of the formation and configuration of the network, network resource management, network diagnostics and maintenance, and security management. Application sub-layer: complete network time synchronization, node location, and provides user-oriented applications collaborative application service interface. Application layer communication includes information access applications and settings between different devices on the network.

In actual measurement, since the resistance wire strain is very small, in addition to the pressure on the specimen impacts resistance strain gauge, the ambient temperature can change the resistance of the resistance wire, thus affects the overall measurement results, it is necessary to take effective temperature compensation. Industrial applications require protocol provides support for continuous, discrete control different applications. The network layer field devices can support three major topologies: star, mesh, star network type (star and mesh hybrid), through the formation of a variety of flexible topologies can cover wide applications.

\section{Hardware and Software System Design of Wireless Sensor Networks}

The system uses a three-tier network topology tree: The first layer is the gateway is the network coordinator, which is connected with the host computer using the RS232 serial bus, and the other nodes in the network use a wireless network communications; second layer is the routing nodes, a fully functional network node (FFD), which call other network coordinator node at any time; the third layer is the network end nodes, using streamlined functional nodes (RFD), can only communicate with the routing node or network coordinator between the RFD can't communicate ${ }^{[5]}$. Fig. 3 shows the hardware design of wireless sensor networks.

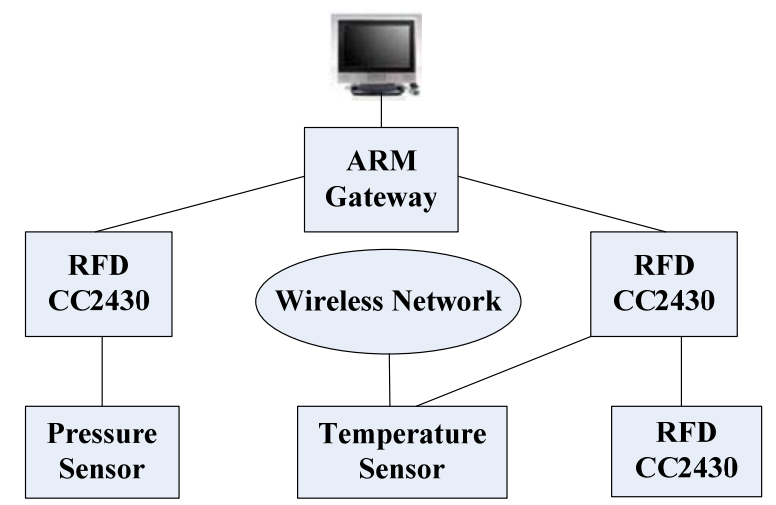

Fig. 3 The hardware design of wireless sensor networks

The system has a network coordinator, a terminal node and three routing nodes. I terminal node 
which is directly connected to communicate with the network coordinator, and the terminal nodes with strain test circuit, the router II node is responsible for III and IV nodes routing functions; III node and IV nodes as I node are RFD nodes ${ }^{[6]}$.

Among them, the whole network is divided into the program coordinator of the network program and a terminal node, the master node main function is started to initialize a network, generate code and PAN broadcast, monitor the entire network, allowing other nodes to meet the conditions to join the network and is responsible for sending command requests and receive data terminal node and the like. When the primary node is powered, first initialize the network, and then begins to initialize the protocol stack and the interrupts are enabled. Then it began to scan the network to produce PAN code, create a new network, and broadcast out, if the network is successfully established, the master node on the LCD screen will display the network ID and channel information. After the master node will enter the monitoring status, see if there is a command from the upper or join the network requests from other nodes, if the request is assigned to the node network address; if the upper sensor read command, the master node will read the corresponding sensor values and data back, and then enter the wireless monitoring state again; if there is no request, the program automatically returns to re-enter the monitoring state.

\section{Conclusions}

In recent years, sensor has a broad application prospects, industry and academia much attention, the article mainly research sensors in industrial networks. Studies have shown that the use of sensor networks in industrial networks for monitoring and control, without wiring restriction can reduce installation and maintenance costs, provide better system performance; there are still a number of key technologies to be resolved, wireless sensor network technology matures and actual deployment will greatly promote the development of industrial networks. This article discusses the sensor network applications in industrial networks, industrial network environment, there are a number of key technologies to be resolved, it is certain that once the future of wireless sensor networks are in energy efficiency, fault tolerance, routing management, improvement to meet industrial networking reliability and real-time response requirements, sensor networks widely deployed in industry, industrial production has played a huge role in promoting.

\section{References}

[1] Yuan R. L. et al. Health Monitoring of Light-Rail Aerial-Structural System[C]. NDE for Health Monitoring and Diagnostics, San Diego, 2002.

[2] ZHEN G J ,L EE M J .A Comprehensive Performance Study of IEEE802. 1 S. 4 [M]. IEEE Press Book, 2004.

[3] M. Antoniou, M. C. Boon, P. N. Green, P. P. Green and T. A. York. Wireless sensor networks for industrial processes. IEEE Sensors Applications Symposium [C]. 2009.

[4] M. Hanssmann, S. Rhee, and S. Liu. No warning constraints [J]. IEEE Industry Applications Magazine, 2009.

[5] J. Song, A. K. Mok, D. Chen, and M. Nixon. Workshop Challenges of wireless control in process Industry. Research on Directions for Security and Networking in Critical Real Time and Embedded Systems [C]. 2006. 